\section{Registros em saúde: avaliação da qualidade do prontuário do paciente na atenção básica, Rio de Janeiro, Brasil}

\author{
Health records: evaluation of patient health charts \\ in primary care, Rio de Janeiro, Brazil
}

\author{
${ }_{1}$ Escola Nacional de Saúde \\ Pública Sergio Arouca, \\ Fundação Oswaldo Cruz, Rio \\ de Janeiro, Brasil. \\ Correspondência \\ M. M. Vasconcellos \\ Departamento de \\ Administração e \\ Planejamento em Saúde, \\ Escola Nacional de Saúde \\ Pública Sergio Arouca, \\ Fundação Oswaldo Cruz. \\ Rua Leopoldo Bulhões 1480 \\ sala 727 , Rio de Janeiro, $R J$ \\ 21041-210, Brasil. \\ miguel@ensp.fiocruz.br
}

\begin{abstract}
With the purpose of contributing to the evaluation of primary care, a study was conducted based on the quality of patients' health charts, considering the records for care provided in $4 \mathrm{mu}$ nicipalities with more than 100 thousand inhabitants each in the State of Rio de Janeiro, Brazil, in 2004. This was a cross-sectional study based on primary data collected from direct consultation of patient charts. A two-stage, probabilistic cluster sample was selected from primary care facilities and consultations/patient charts. We checked the completeness of attributes pertaining to the identification of users receiving care and the characteristics of the care itself (weight, blood pressure, Pap tests, and blood glucose) on the charts of women over 19 years of age with hypertension and/or diabetes. User identification showed a low presence of social attributes, and only half of the charts had recorded the opening date. Records of process characteristics in care provided to women with hypertension and diabetes failed to conform to Brazilian Ministry of Health guidelines. Analysis of completeness suggests dubious quality in the continuity of the care provided and difficulties in management practice for primary care and implementation of the Family Health Strategy.
\end{abstract}

Primary Health Care; Quality of Health Care; Health Services Evaluation; Medical Records
Miguel Murat Vasconcellos 1

Else Bartholdy Gribel 1

Ilara Hammerli Sozzi de Moraes 1

\section{Introdução}

A importância do registro em saúde teve seu início na prática clínica, na qual os profissionais de saúde identificaram a necessidade de recorrer à história clínica e evolutiva para acompanhamento dos doentes 1 . Estratégico para a decisão clínica e gerencial, para o apoio à pesquisa e formação profissional, atualmente o registro é considerado critério de avaliação da qualidade da prestação de serviço de saúde, isto é, a qualidade dos registros efetuados é reflexo da qualidade da assistência prestada, sendo ponto chave para informar acerca do processo de trabalho 2 .

Tratado aqui como registro em saúde, prontuário é definido como o documento único, constituído de um conjunto de informações, sinais e imagens registradas, geradas com base em fatos, acontecimentos e situações sobre a saúde do paciente e a assistência a ele prestada, de caráter legal, sigiloso e científico, que possibilita a comunicação entre membros da equipe multiprofissional e a continuidade da assistência prestada ao indivíduo 3,4. Destina-se, ainda, ao subsídio dos processos de gestão, ao ensino e à pesquisa em saúde; à formulação, implementação e avaliação de políticas públicas; além de documentar demandas legais. Os usuários dos prontuários são, portanto, os profissionais diretamente envolvidos na prestação do cuidado e na gestão, pesquisadores, docentes, alunos do campo da saúde e o próprio paciente. Todos os 
registros preenchidos e organizados de forma a permitir sua localização, leitura e identificação de autoria deverão ser completos, acurados, objetivos e concisos ${ }^{5}$. Dessa forma, potencializam o uso de bases de dados administrativas, que têm prontuários como fonte de dados, na avaliação da qualidade da assistência à saúde 6 .

Apesar dessa importância, no Brasil, a literatura vem apontando baixa qualidade do registro em saúde. Nos anos 1980, Lotufo \& Duarte 7, tratando da programação local em Mato Grosso, constataram elevada freqüência de diagnósticos ilegíveis, incoerências de registros e ausência de diagnóstico. Da mesma forma, Modesto et al. 8 observaram elevada proporção (76\%) de prontuários que não possuíam itens considerados básicos, ao analisarem a Reforma Sanitária na Bahia, por intermédio da qualidade dos registros médicos. Nos anos 1990, Scochi ${ }^{9}$, estudando a qualidade dos prontuários em serviços ambulatoriais no Paraná, apontou seu funcionamento precário como fonte de informação, tanto para acompanhamento dos pacientes como para avaliação dos serviços. Munhoz Jr. et al. 10 observaram registro inadequado das informações dos pacientes em mais da metade dos prontuários analisados, avaliando o Programa de controle da hanseníase no Mato Grosso do Sul.

No Brasil, a principal estratégia proposta pela esfera federal para organização e fortalecimento da Atenção Básica no Brasil é o Projeto de Expansão e Consolidação do Saúde da Família (PROESF) que pretende "a operacionalização de um sistema de atenção hegemônico apoiado em princípios e características do Saúde da Família, conceitos de risco social e epidemiológico, noção de território, fortalecimento de vínculos entre população e serviços, transformação dos processos de trabalho e nas práticas dos profissionais de saúde, ações intersetoriais e de promoção de saúde, além da introdução de sistemas de avaliação e monitoramento" 11 (p. 7). Em relação à Atenção Primária à Saúde, Novaes 12 observou que, na literatura nacional, a abordagem predominante se dá na perspectiva de programas específicos em que ora se enfatizam dimensões de gestão e financiamento, ora se analisam alguns indicadores gerais de desempenho do programa em uma perspectiva populacional, geralmente sem detalhamento das características dos serviços e da assistência realizada.

Quando trata de registros na Atenção Primária à Saúde, de forma um pouco diferenciada da nacional, a literatura internacional vem privilegiando análises da associação do Registro em Saúde, em formato eletrônico, em relação à qualidade de dados do próprio registro, do cuidado prestado ou ainda da gestão da assistência.
Heymann et al. 13 sugerem a estruturação do registro eletrônico em saúde com campos obrigatórios para melhorar a efetividade de screening de pressão arterial. Thiru et al. ${ }^{14}$, fazendo revisão sistemática do escopo e da qualidade de dados dos registros eletrônicos e na atenção primária em saúde, ponderam que a falta de padrões dificulta a comparação dos resultados entre os estudos analisados e apontam a necessidade de adoção de medidas claras para análise dessa qualidade. Outra revisão da literatura sobre melhoria da qualidade de dados na Atenção Primária à Saúde 15 encontrou poucos estudos e, também, ausência de padrões de métodos de análise, sugerindo que o empenho na completitude do registro parece ser o primeiro passo na melhoria de sua qualidade. Sem vinculação com a perspectiva eletrônica, Lauteslager et al. ${ }^{16}$ sugerem a incorporação do paciente como fonte para melhoria do registro em saúde.

Ao contrário de Adams et al. 17 que, estudando centros de saúde pediátricos, relataram associação entre uso de registro em saúde com melhoria da qualidade do cuidado, Linder et al. 18, mesmo considerando que tecnologias de informação e registro eletrônico em saúde possam melhorar a qualidade da atenção, não encontraram associação entre registro eletrônico em saúde e melhoria da qualidade na atenção ambulatorial. De outro lado, Wager et al. 19, estudando o impacto da implementação de registro eletrônico em saúde em clínicas de Atenção Primária à Saúde, sugerem que ocorrem mudanças não só no gerenciamento do prontuário do paciente mas também na estrutura organizacional e como os médicos se comunicam entre si e com os provedores de serviços. Para Welch et al. 20 é necessário mais pesquisas para entender as nuances da implementação de registro eletrônico em saúde em unidades de Atenção Primária à Saúde, pois o impacto de introdução de novas tecnologias em processos de trabalho complexos ainda não está bem esclarecido e não pode ser automaticamente equiparado tanto para a melhoria da qualidade do cuidado, quanto para a diminuição dos custos.

Bodenheimer \& Grumbach ${ }^{21}$ sugerem que a tecnologia eletrônica tem um grande potencial para aperfeiçoar a Atenção Primária à Saúde em áreas do registro em saúde, comunicação entre médicos e pacientes, compartilhamento de informação entre prestadores de serviços de saúde e rápido acesso à informação médica confiável. No entanto, assim como afirma Majeed 22, para que isso aconteça é preciso superar barreiras como quantidade de tempo e custos da implementação, a inadequação da informação médica com base na Internet, a falta de padrões, a necessida- 
de de proteção da privacidade do paciente e a possibilidade de interferência, pelo computador, na relação médico-paciente.

Diante desse quadro, este artigo pretende contribuir para o avanço tanto do processo de trabalho, quanto do processo avaliativo de serviços básicos em saúde, ao analisar características dos registros dos atendimentos realizados por esses serviços, considerando a completitude de componentes fundamentais do prontuário do paciente.

\section{Materiais e método}

Inserido no contexto da pesquisa Desenvolvimento de Estudo de Linhas de Base nos Municípios Selecionados do Projeto de Expansão e Consolidação da Saúde da Família - Região Sudeste 2 (ELB-PROESF) 23, que avaliou a Atenção Básica de Saúde nos 22 municípios de mais de 100 mil habitantes no Estado do Rio de Janeiro, o presente artigo consiste em estudo transversal com base em dados primários, levantados mediante verificação direta no prontuário por pesquisador treinado especificamente para tal fim. De forma articulada com dados de inquérito em estabelecimentos de saúde 23 , foram analisados os registros dos atendimentos de profissionais de saúde de nível superior realizados em 2004, em quatro municípios. Selecionados como objetos de estudo de caso, esses municípios foram escolhidos por suas características singulares, quase sempre distintas, quanto ao contexto político, porte populacional, renda per capita, cobertura do Programa Saúde da Família (PSF), Índice de Desenvolvimento Humano Municipal (IDH-M) e à estrutura assistencial na atenção básica. A Tabela 1 detalha esses indicadores, alguns com dados aproximados para que o município não seja identificado, além de apontar os municípios 1 e 2 como aqueles com maiores indicações de pobreza e os municípios 3 e 4 como aqueles com maiores indicações de riqueza.

Tendo como base o delineamento amostral definido por Silva \& Reis 24 e conceitos tratados por Cochran 25, os atendimentos foram sorteados em amostra probabilística por conglomerado em dois estágios de estabelecimentos e atendimentos/prontuários em cada um dos quatro municípios. O primeiro estágio referiu-se aos estabelecimentos de saúde da atenção básica (unidades primárias de amostragem) e o segundo estágio consistiu nos atendimentos realizados por profissional de saúde de nível superior nesses estabelecimentos, em 2004, junto com os prontuários em que tais atendimentos foram registrados.
O universo de estabelecimentos foi obtido no Cadastro Nacional de Estabelecimentos de Saúde (CNES), considerando-se aqueles classificados como ambulatoriais. A lista dos estabelecimentos foi submetida às equipes gestoras das Secretarias Municipais de Saúde para confirmação da existência e definição daqueles estabelecimentos como inseridos na atenção básica. Assim, foram feitas as adaptações para definição final do universo de estabelecimentos da atenção básica em cada um dos quatro estudos de caso. Os estabelecimentos foram selecionados aleatoriamente, de forma proporcional ao número existente em cada um dos municípios, assegurando-se o número mínimo de trinta estabelecimentos em cada município. Adotando-se um intervalo de 95\% de confiança e um erro máximo de amostragem de $15 \%$, obteve-se uma amostra de 122 estabelecimentos (Tabela 1).

O tamanho mínimo da amostra de atendimentos foi de 500 atendimentos em cada município, determinado pelos mesmos critérios para o tamanho da amostra de estabelecimentos e modificando-se o erro máximo de amostragem para 5\%. Os atendimentos realizados em 2004 foram listados para cada estabelecimento sorteado. A seguir, foram selecionados sistematicamente vinte atendimentos em cada um dos estabelecimentos amostrados. Portanto, os achados relativos às condições de registro dos atendimentos são representativos para cada um dos municípios e não para cada estabelecimento.

Foram analisados 2.291 registros de atendimentos realizados nos 4 municípios e seus prontuários. Dos atendimentos sorteados não foram encontrados 149 prontuários correspondentes nos estabelecimentos de saúde que estavam distribuídos de maneira praticamente uniforme entre os quatro municípios (Tabela 1).

Diante da dificuldade em listar os atendimentos realizados em 2004, em cada estabelecimento, necessário para o sorteio sistemático dos atendimentos, optou-se por utilizar as planilhas diárias em que os profissionais de saúde de nível superior listam os atendimentos realizados. Tais planilhas vão constituir o Boletim de Produção Ambulatorial e o Sistema de Informação Ambulatorial do Sistema Único de Saúde (SIA/SUS). Preenchidas manualmente pelos profissionais individualmente, as planilhas estão disponíveis por município, por estabelecimento, por profissional, por data e contêm os nomes dos pacientes atendidos (Secretaria de Estado de Saúde do Rio de Janeiro. http://www.saude.rj.gov.br, acessado em 20/Jan/2005). Permitiram gerar as listas dos atendimentos que constituíram a base para o cálculo do intervalo aleatório dos atendimentos e do número aleatório inicial. Também permitiram a 
Características censitárias, sócio-demográficas, número de estabelecimentos da atenção básica e atendimentos pesquisados, em quatro estudos de caso. Estado do Rio de Janeiro, Brasil, 2004

\begin{tabular}{|c|c|c|c|c|}
\hline Características & Município 1 & Município 2 & Município 3 & Município 4 \\
\hline População (mil) * & 750 & 200 & 450 & 250 \\
\hline$\%$ analfabetos no grupo de $18-24$ anos & 2,4 & 2,9 & 1,1 & 1,0 \\
\hline Renda per capita & 226,1 & 202,3 & 809,2 & 348,2 \\
\hline Cobertura PSF (2004) ** & 13,2 & 71,9 & 65,8 & 30,5 \\
\hline 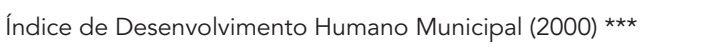 & 0,75 & 0,73 & 0,88 & 0,81 \\
\hline Despesas totais em saúde por habitante $(R \$)$ ** & 145,00 & 148,00 & 301,00 & 267,00 \\
\hline Número de unidades públicas de atenção básica ** & 153 & 72 & 50 & 60 \\
\hline Número de unidades públicas de atenção básica amostradas ** & 30 & 31 & 30 & 31 \\
\hline Número de atendimentos amostrados ** & 580 & 568 & 568 & 575 \\
\hline
\end{tabular}

PSF: Programa Saúde da Família.

* Instituto Brasileiro de Geografia e Estatística (http://www.ibge.gov.br);

** Mendonça et al. 23;

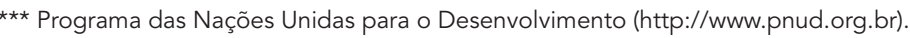

identificação do usuário que levou à localização, nos estabelecimentos de saúde, dos prontuários correspondentes. Com base na listagem com o nome do usuário, profissional de saúde e data do atendimento, fornecida pelos pesquisadores de campo, os funcionários dos estabelecimentos de saúde separaram os prontuários. Em alguns casos foi feita busca em todos os prontuários, pois sendo a lógica de armazenamento por família e/ ou por endereço, não foi possível localizar diretamente o prontuário pelo nome do usuário. Também foram encontrados estabelecimentos que armazenavam prontuários por programa, apesar de seus gerentes ou responsáveis relatarem utilizar prontuário único, sendo necessário recuperar as partes em mais de um arquivo.

O Inquérito em Estabelecimentos de Saúde teve como referência principal a caracterização gerencial elaborada por Lima et al. 26, adaptada para serviços básicos, além de proposta de monitoramento do Ministério da Saúde 27. Os responsáveis pelos estabelecimentos do primeiro estágio da amostra responderam a questionário fechado, aplicado pelo mesmo pesquisador que analisou os prontuários, abordando diversos aspectos como a relação com o território onde está localizado e com a rede de serviços de saúde, capacidade instalada, modelo organizacional, práticas de saúde e qualidade assistencial. Tal inquérito deu origem aos dados detalhados na Tabela 2 e sobre a existência de comissão de avaliação ou de revisão de prontuários utilizados como contraponto de indicadores de perspectiva gerencial.

\section{Indicadores de avaliação e classificações utilizados}

Entre os critérios propostos por Romero \& Cunha ${ }^{28}$ para avaliar a qualidade das variáveis do Sistema de Informações sobre Mortalidade (SIM) encontra-se a completitude, medida pela proporção de informação ignorada no preenchimento de determinada variável. Criaram um escore com os seguintes graus de avaliação: excelente (menor de $5 \%)$, bom (5\%-10\%), regular (11\%-20\%), ruim (21\%-50\%) e muito ruim (mais de $50 \%$ ).

De forma análoga, para efeito de análise no presente trabalho, foi considerada a presença dos atributos nos prontuários, conforme Resolução do Conselho Federal de Medicina 4, que define prontuário médico, e Portaria GM no . 3.947/9829, do Ministério da Saúde, que define os atributos comuns a serem adotados, obrigatoriamente, por todos os sistemas de informações de saúde. Assim, para a caracterização da completitude da identificação do prontuário e do indivíduo atendido foram considerados os seguintes atributos: nome completo; data de nascimento; sexo; naturalidade; endereço completo; data de abertura do prontuário; situação familiar (vive só, convive somente com esposa, convive com esposa e filhos, entre outras); escolaridade e ocupação (situação no mercado de trabalho).

Em relação à completitude de características do processo de atendimento foram selecionados aqueles realizados em mulheres acima de 19 anos e em portadores de hipertensão arterial e/ou diabetes mellitus. Nos primeiros foi considerada a presença, no registro do atendimento sorteado 
Características da atenção básica, de exames ginecológicos e de condições do setor de arquivo de prontuários, em quatro estudos de caso. Estado do Rio de Janeiro, Brasil, 2004

\begin{tabular}{|c|c|c|c|c|}
\hline Características & Município 1 & Município 2 & Município 3 & Município 4 \\
\hline \% de estabelecimentos que relataram operar com população adscrita & 80,0 & 80,6 & 93,3 & 77,4 \\
\hline \% de estabelecimentos com capacidade para informar população residente & 3,3 & 3,2 & 36,7 & 67,7 \\
\hline \% de estabelecimentos com capacidade para informar população cadastrada & 23,3 & 6,5 & 30,0 & 25,8 \\
\hline \% de mulheres que relataram exame ginecológico nos últimos três anos & 86,8 & 81,8 & 88,3 & 83,4 \\
\hline \% de estabelecimentos com mobiliário do setor de arquivo de prontuários adequado & 36,7 & 19,4 & 30,0 & 20,0 \\
\hline
\end{tabular}

Fonte: Mendonça et al. 23

e nos três atendimentos anteriores, caso existissem, do peso, da pressão arterial, da realização e do resultado do exame colpocitológico (Papanicolaou). No caso da colpocitologia, o registro tanto da realização, quanto do resultado, teve sua presença verificada em toda extensão do prontuário. Nos prontuários dos hipertensos e/ou diabéticos foi verificada a presença, no registro do atendimento sorteado e nos três atendimentos anteriores, caso existissem, do peso, da pressão arterial, da glicemia e do perímetro da cintura.

A seleção dos prontuários de mulheres acima de 19 anos deve-se à utilização, pelo ELB-PROESF, de condições marcadoras como um dos métodos de avaliação ${ }^{30}$. Entre elas destaca-se o controle do câncer de colo uterino, abordado sob diversas perspectivas nos instrumentos aplicados, sendo enfatizada a análise das ações desenvolvidas no âmbito da Atenção Básica. O Ministério da Saúde recomenda que a periodicidade para realização do preventivo ginecológico seja de três anos 31 . A relevância da análise dos prontuários dos pacientes hipertensos e diabéticos justifica-se pelas doenças cardiovasculares constituírem-se na principal causa de morbimortalidade na população brasileira. A hipertensão arterial e o diabetes são dois dos principais fatores de risco para essas doenças. Para acompanhamento dos portadores de hipertensão arterial e diabetes, o Ministério da Saúde 32,33 preconiza a verificação e registro da pressão arterial, peso, altura e glicemia em todos os atendimentos.

Dessa forma, no município 1 foi realizada análise de 580 atendimentos: 313 foram atendimentos em mulheres acima de 19 anos e 163 em pessoas portadoras de hipertensão arterial e/ou diabetes. No município 2, dos 568 atendimentos levantados, 313 eram referidos a mulheres acima de 19 anos e 183 foram relativos a pessoas portadoras de hipertensão arterial e/ou diabetes. No município 3, foram analisados 568 atendimen- tos, sendo 278 realizados em mulheres acima de 19 anos e 125 em pessoas portadoras de hipertensão arterial e/ou diabetes. No município 4, foram analisados 575 atendimentos, sendo 226 realizados em mulheres acima de 19 anos e 113 em pessoas portadoras de hipertensão arterial e/ou diabetes.

Este estudo foi apresentado ao Comitê de Ética da Escola Nacional de Saúde Pública Sergio Arouca, Fundação Oswaldo Cruz, com parecer favorável no. 23/05 - CAAE no 0075.0.031. 000-05.

\section{Resultados}

Neste trabalho a análise dos dados foi realizada de duas perspectivas. A primeira tratou da completitude da identificação do indivíduo nos prontuários correspondentes aos atendimentos sorteados. A segunda perspectiva considerou algumas características do processo de atendimento registradas no prontuário. Realizou-se, ainda, análise comparativa entre os quatro municípios e articulações com resultados do Inquérito em Estabelecimentos de Saúde do ELB-PROESF.

Detalhada na Tabela 3, a avaliação da completitude da identificação nos prontuários apontou, segundo a classificação de Romero \& Cunha 28 , presença do nome do usuário entre excelente $\mathrm{e}$ bom nos quatro municípios. Dois municípios (1 e 4) apresentaram registro apenas regular do endereço completo. O registro da data de abertura do prontuário mostrou-se ruim e muito ruim nos municípios 1, 3 e 4 e apenas regular no município 2. Quanto ao registro do sexo, os municípios 1 e 4 classificaram-se como ruim, o município 3 como regular e, somente o município 2 pode ser classificado como bom.

Foi verificado registro muito ruim dos atributos sociais como situação familiar, escolaridade 
Percentual de atendimentos, segundo presença de registro de atributos de identificação do indivíduo nos prontuários dos atendimentos sorteados, em quatro estudos de caso. Estado do Rio de Janeiro, Brasil, 2004.

\begin{tabular}{|c|c|c|c|c|}
\hline Atributos & Município 1 (\%) & Município 2 (\%) & Município 3 (\%) & Município 4 (\%) \\
\hline Nome completo & 91,2 & 100,0 & 98,8 & 98,6 \\
\hline Data de abertura & 60,9 & 87,7 & 16,7 & 37,8 \\
\hline Endereço completo & 87,9 & 96,7 & 91,5 & 89,9 \\
\hline Data de nascimento & 75,5 & 97,7 & 97,2 & 98,8 \\
\hline Sexo & 60,5 & 94,4 & 86,8 & 61,6 \\
\hline Naturalidade & 48,4 & 81,5 & 68,1 & 55,9 \\
\hline Situação familiar & 49,8 & 5,1 & 34,2 & 5,2 \\
\hline Escolaridade & 76,0 & 1,4 & 32,4 & 10,8 \\
\hline Ocupação (situação no mercado de trabalho) & 65,0 & 68,0 & 36,1 & 34,7 \\
\hline
\end{tabular}

Fonte: Mendonça et al. 23.

e ocupação nos prontuários de todos os quatro municípios. Foram encontrados percentuais de presença extremamente baixos no registro da escolaridade $(1,4 \%)$ e situação familiar $(5,1 \%)$ no município 2.

Em relação às características do processo de atendimento registradas nos prontuários das mulheres acima de 19 anos (Tabela 4) como peso, pressão arterial, realização e resultado da colpocitologia, somente uma delas (realização do exame colpocitológico), mesmo assim em apenas dois municípios, foi registrada em pouco mais da metade dos atendimentos. Todas as outras importantes características mostraram presença de seus registros classificada como muito ruim. Em relação à anotação da realização e do resultado do exame colpocitológico deve ser notado que sua verificação foi realizada em todo prontuário, pelo pesquisador de campo, e não apenas no atendimento sorteado.

Referentemente às características do processo de atendimento registradas nos prontuários dos portadores de hipertensão e/ou diabetes (Tabela 5), observou-se que o registro do peso, cuja verificação é preconizada em todas as consultas ${ }^{32}$, é muito ruim, com todos os municípios apresentando completitude abaixo de 50\%. Note-se que, em dois deles, o não-registro ocorre em torno de $90 \%$ dos atendimentos. A completitude do registro da pressão arterial, apesar de ser maior do que no caso do peso, é ruim em todos os municípios, pois se espera que $100 \%$ dos usuários tenham sua aferição e registro no momento da consulta. O registro da glicemia é muito ruim de forma homogênea nos quatro municípios. $\mathrm{O}$ registro da verificação do perímetro da cintura é praticamente inexistente nos municípios pes- quisados, ainda que seja um sinal importante na continuidade do cuidado prestado tanto ao paciente hipertenso quanto ao diabético.

A Tabela 2 detalha indicadores construídos mediante inquérito em estabelecimentos de saúde e do inquérito domiciliar, do ELB-PROESF, selecionados para análise complementar neste estudo 23 . Os dois primeiros referem-se à capacidade dos estabelecimentos da amostra de trabalharem com população adscrita. É preciso levar em conta a possibilidade de influência da cobertura do PSF, em cada município, por privilegiar o vínculo entre a equipe de saúde e a população coberta. Embora grande parte dos responsáveis pelos estabelecimentos da amostra relate operar com população adscrita em todos os municípios estudados, somente o município 4 apresentou percentual de estabelecimentos acima de 50\%, cujos responsáveis ou gerentes foram capazes de informar a quantidade de pessoas residentes no território sob sua responsabilidade. Entretanto, o maior percentual de estabelecimentos cujos responsáveis ou gerentes foram capazes de informar a quantidade de pessoas cadastradas não passou de $30 \%$ e foi encontrado no município 3. De outro lado, chama a atenção que em somente $6,5 \%$ dos estabelecimentos do município 2 foi possível obter informação sobre o total da população cadastrada. O inquérito domiciliar mostrou a grande adesão das mulheres à realização do exame preventivo do câncer cérvico-uterino (em torno de $90 \%$ daquelas que fizeram exame ginecológico) em todos os quatro municípios. Finalmente, em torno de $70 \%$ dos estabelecimentos pesquisados tiveram o mobiliário do setor de arquivo de prontuários considerados inadequados. 
Percentual de atendimentos, segundo condição da presença de características do processo de atendimento em mulheres acima de 19 anos, em quatro estudos de caso. Estado do Rio de Janeiro, Brasil, 2004

\begin{tabular}{|c|c|c|c|c|}
\hline Atributos & Município 1 (\%) & Município 2 (\%) & Município 3 (\%) & Município 4 (\%) \\
\hline Peso & 23,0 & 11,2 & 34,5 & 9,3 \\
\hline Pressão arterial & 49,5 & 39,6 & 42,8 & 44,9 \\
\hline Realização de colpocitologia & 45,7 & 51,8 & 54,7 & 34,8 \\
\hline Resultado do exame colpocitológico & 38,0 & 37,7 & 36,3 & 24,7 \\
\hline
\end{tabular}

Fonte: Mendonça et al. 23.

Tabela 5

Percentual de atendimentos, segundo condição da presença de características do processo de atendimento em portadores de hipertensão e diabetes, em quatro estudos de caso. Estado do Rio de Janeiro, Brasil, 2004.

\begin{tabular}{|c|c|c|c|c|}
\hline Atributos & Município 1 (\%) & Município 2 (\%) & Município 3 (\%) & Município 4 (\%) \\
\hline Peso & 24,5 & 12,0 & 40,8 & 8,8 \\
\hline Pressão arterial & 73,0 & 58,5 & 60,0 & 77,9 \\
\hline Glicemia & 18,4 & 10,9 & 8,0 & 11,5 \\
\hline Perímetro da cintura & 1,2 & 2,7 & 0,0 & 0,9 \\
\hline
\end{tabular}

Fonte: Mendonça et al. 23.

\section{Discussão}

Evidentemente não se pretende uma análise do desempenho do sistema de saúde dos municípios analisados na medida em que seria necessária a utilização de matriz de indicadores complexa, comosugereaPRO-ADESS:MetodologiadeAvaliação do Desempenho do Sistema de Saúde Brasileiro ${ }^{34}$. No entanto, mesmo considerando suas possíveis limitações, a análise dos dados permite discussões de três perspectivas: da clínica ou do cuidado prestado, da gestão dos serviços de Atenção Básica e da própria Estratégia da Saúde da Família.

Supondo que a qualidade dos registros efetuados seja reflexo da qualidade da assistência prestada e que os registros médicos são fundamentais para informar acerca do processo de trabalho, como considera Donabedian 2, os achados do presente estudo apontam para uma prática clínica e do cuidado prestado precários em todos os municípios estudados. Tal precariedade independe das características do município, seja o valor do IDH-M, seja o tamanho da população ou a extensão da cobertura do PSF. A pequena presença de registro de características do processo de atendimento na amostra, como peso, pressão arterial ou glicemia sugere dificuldades na continuidade da prestação do cuidado e baixa qualidade técnico-científica. Note-se que essa continuidade relaciona-se a arranjos dos serviços para que a atenção oferecida apresente uma sucessão ininterrupta de eventos, incluindo mecanismos que caracterizam o prosseguimento da assistência, como o registro em prontuários 35, e que aquela qualidade técnicocientífica corresponde à adequação das ações ao conhecimento vigente. Registre-se que somente dez estabelecimentos informaram possuir comissão de avaliação ou revisão de prontuário, sendo oito deles no município 3 e dois localizados no município 423 .

Além disso, o baixo registro da data de abertura nos prontuários analisados impediu a identificação dos prontuários abertos recentemente, prejudicando uma análise mais ampla da continuidade do cuidado, conforme preconizado nos protocolos dos programas do Ministério da Saúde. Desse modo, não foi possível analisar a data dos três últimos atendimentos realizados e as condições do registro de suas características, coletadas na aplicação do instrumento.

O registro ruim e muito ruim nos municípios 1, 3 e 4 e apenas regular no município 2 da data 
de abertura do prontuário sugere problemas na estruturação de processos de trabalho nos estabelecimentos da amostra. De outro lado, determinou dificuldades para o estudo da periodicidade do atendimento à população objeto de programas de saúde, como mulheres em idade fértil e portadores de doenças crônicas. Da mesma forma, dificulta o uso de instrumentos gerenciais pelos responsáveis dos estabelecimentos de saúde estudados para geração de indicadores, com base no prontuário, no acompanhamento de metas das ações dos programas de saúde. A essas dificuldades na utilização de instrumentos gerenciais ou da própria gestão dos serviços de saúde da Atenção Básica devem ser acrescentados problemas na operação com o conceito de população adscrita.

Os responsáveis pelos estabelecimentos pesquisados tiveram dificuldades de quantificar tanto os dados relativos à população residente, quanto dados relativos à população cadastrada em seu território de abrangência. Como o registro das características do processo de atendimento pesquisadas (por exemplo: registro da realização da colpocitologia) foi muito ruim, cabe levantar a hipótese de que os estabelecimentos pesquisados não trabalham com acompanhamento de metas de cobertura. Tal fato ocorre em qualquer um dos municípios pesquisados, independente da cobertura de implementação da Estratégia Saúde da Família. Chama a atenção que o inquérito domiciliar do ELB-PROESF mostrou grande adesão das mulheres à realização do exame preventivo do câncer cérvico-uterino (maior de $80 \%$ em cada um dos 4 municípios 30 ) em con-

\section{Resumo}

Com o objetivo de contribuir para a avaliação da Atenção Básica, desenvolveu-se estudo que tem por referência a qualidade de prontuários dos pacientes, considerando os registros dos atendimentos realizados em quatro municípios acima de 100 mil habitantes no Estado do Rio de Janeiro, Brasil, em 2004, esta pesquisa se trata de estudo transversal com base em dados primários, levantados por verificação direta no prontuário. Os atendimentos foram sorteados em amostra probabilística por conglomerado em dois estágios: estabelecimentos de atenção básica e atendimentos/ prontuários. Verificou-se a completitude de atributos relativos à identificação dos usuários atendidos, em todos os prontuários, e a características do processo de atendimento (como peso, pressão arterial, colpocito- traste com o relativamente baixo registro tanto da realização, quanto do resultado desse exame no prontuário. Ainda em relação aos aspectos gerenciais, dois municípios (1 e 4) apresentaram registro apenas regular do endereço completo, fato que, certamente, prejudica os processos de busca ativa de casos, monitoramento de ações de programas de saúde e visitação domiciliar.

Deve ser notada a pequena presença do registro das condições de situação familiar que, além de ser variável fundamental também para acompanhamento clínico individual, é estratégica em áreas de atuação de programas que têm a família como objeto. Considerando a análise dos dados apresentados pelos estabelecimentos de saúde da amostra, transformações como adoção na práxis de conceitos de risco epidemiológico, território, fortalecimento de vínculos entre população e serviços, inovações nos processos de trabalho e nas práticas dos profissionais de saúde, pretendidas pela Estratégia Saúde da Família parecem ainda distantes de se efetivarem.

A melhoria da qualidade do registro em saúde, mediante incentivo à sua estruturação com campos obrigatórios 2,13,21, de forma padronizada, com ou sem a incorporação de sistemas eletrônicos, tem um potencial inequívoco como infra-estrutura necessária para uma adequada atenção à saúde e uma organização dos serviços capaz de aproximar-se da integralidade do cuidado, com eqüidade e acesso universal.

A discussão dos resultados com os gestores de saúde vem possibilitando a compreensão mais clara dos problemas encontrados e o desenho de possíveis soluções para sua superação. logia e glicemia) nos prontuários de mulheres acima de 19 anos, hipertensas e diabéticas. Na identificação dos usuários, encontrou-se baixa presença dos atributos sociais e somente metade dos prontuários possuía registro da data de abertura. Os registros de características do processo de atendimento em mulheres, hipertensas e diabéticas, ficaram longe das proposições do Ministério da Saúde. A análise da completitude sugere discutível qualidade na continuidade do cuidado oferecido, dificuldades para a prática gerencial na Atenção Básica e para implementação da Estratégia Saúde da Família.

Atenção Primária à Saúde; Qualidade da Assistência à Saúde; Avaliação de Serviços de Saúde; Registros Médicos 


\section{Colaboradores}

M. M. Vasconcellos e E. B. Gribel contribuíram com o desenho do estudo, a coordenação do trabalho de campo, análise dos dados, discussão dos resultados e redação do texto. I. H. S. Moraes contribuiu na análise dos dados, discussão dos resultados e redação do texto.

\section{Referências}

1. Moraes IHS. Política, tecnologia e informação em saúde - a utopia da emancipação. Salvador: Instituto de Saúde Coletiva, Universidade Federal da Bahia/Casa da Qualidade Editora; 2002.

2. Donabedian A. The quality of care. How can it be assessed? JAMA 1988; 260:1743-8.

3. Oselka G. Prontuário médico. Rev Assoc Med Bras 2002; 48:286.

4. Conselho Federal de Medicina. Resolução CFM no. 1.638, de 10 de julho de 2002. Define prontuário médico e torna obrigatória a criação da Comissão de Prontuário nas instituições de saúde. Diário Oficial da União 2002; 9 ago.

5. Escola Politécnica de Saúde Joaquim Venâncio. Texto de apoio em registros de saúde. Rio de Janeiro: Editora Fiocruz; 1999.

6. Wray NP, Ashton CM, Kuykendall DH, Hollingsworth JC. Using administrative databases to evaluate the quality of medical care: a conceptual framework. Soc Sci Med 1995; 40:1707-15.

7. Lotufo M, Duarte EC. Avaliação dos serviços de saúde do Município de Cáceres, MT (Brasil): contribuições à programação local. Rev Saúde Pública 1987; 21:427-38.

8. Modesto MG, Moreira EC, Almeida-Filho N. Reforma sanitária e informação em saúde: avaliação dos registros médicos em um distrito sanitário de Salvador, Bahia. Cad Saúde Pública 1992; 8:62-8.

9. Scochi MJ. Indicadores da qualidade dos registros e da assistência ambulatorial em Maringá, (Estado do Paraná, Brasil), 1991: um exercício de avaliação. Cad Saúde Pública 1994; 10:356-67.

10. Munhoz Jr. S, Fontes JF, Meirelles SM. Avaliação do programa de controle da hanseníase em municípios mato-grossenses, Brasil. Rev Saúde Pública 1997; 31:282-7.

11. Dain S. A reestruturação do modelo assistencial de saúde em grandes cidades: padrões de custo e formas de financiamento. Brasília: Secretaria de Políticas de Saúde, Ministério da Saúde; 2002.
12. Novaes HMD. Pesquisa em, sobre e para os serviços de saúde: panorama internacional e questões para a pesquisa em saúde no Brasil. Cad Saúde Pública 2004; 20 Suppl 2:S147-57.

13. Heymann AD, Hoch I, Valinsky L, Shalev V, Silber H, Kokia E. Mandatory computer field for blood pressure measurement improves screening. Fam Pract 2005; 22:168-9.

14. Thiru K, Hassey A, Sullivan F. Systematic review of scope and quality of electronic patient record data in primary care. BMJ 2003; 326:1070-2.

15. Brouwer H, Bindels P, Weert H. Data quality improvement in general practice. Fam Pract 2006; 23:529-36.

16. Lauteslager M, Brouwer HJ, Mohrs J, Bindels PJE, Grundmeijer HGLM. The pacient as a source to improve the medical record. Fam Pract 2002; 19:167-71.

17. Adams WG, Mann AM, Bauchner H. Use of an electronic medical record improves the quality of urban pediatric primary care. Pediatrics 2003; 111:626-32.

18. Linder JA, Ma J, Bates DW, Middleton B, Stafford RS. Electronic health record use and the quality of ambulatory care in the United States. Arch Intern Med 2007; 167:1400-5.

19. Wager KA, Lee FW, White W, Ward DM, Ornstein SM. Impact of an electronic medical record system on community-based primary care practices. J Am Board Fam Pract 2000; 13:338-48.

20. Welch WP, Bazarko D, Ritten K, Burgess Y, Harmon $R$, Sandy LG. Electronic health records in four community physician practices: impact on quality and cost of care. J Am Med Inform Assoc 2007; 14:320-8.

21. Bodenheimer T, Grumbach K. Electronic technology: a spark to revitalize primary care? JAMA 2003; 290:259-64.

22. Majeed A. Ten ways to improve information technology in the NHS. BMJ 2003; 326:202-5. 
23. Mendonça MHM, Najar AL, Giovanella L, Tavares MFL, Vasconcellos MM. Desenvolvimento de Estudo de Linhas de Base nos municípios selecionados do Projeto de Expansão e Consolidação do Saúde da Família - Região Sudeste 2 (ELB-PROESF). Relatório final. http://www.ensp.fiocruz.br/proesf (acessado em 10/Mar/2007).

24. Silva NN, Reis IM. Delineamento amostral para implantação de um Sistema Nacional de Informações de Demanda Ambulatorial. Rev Saúde Pública $1989 ; 23: 298-306$

25. Cochran WG. Sampling techniques. 3rd Ed. New York: John Wiley and Sons; 1977.

26. Lima SML, Portela MC, Barbosa PR, Vasconcellos MM, Ugá MAD, Leis SVG. Caracterização gerencial dos hospitais filantrópicos no Brasil. Cad Saúde Pública 2004; 20:1249-61.

27. Departamento da Atenção Básica, Secretaria de Atenção à Saúde, Ministério da Saúde. Manual dos indicadores de incentivos municipais do PROESF: orientações gerais para o monitoramento - 2a versão. Brasília: Ministério da Saúde; 2005.

28. Romero DE, Cunha CB. Avaliação da qualidade das variáveis sócio-econômicas e demográficas dos óbitos de crianças menores de um ano registrados no Sistema de Informações sobre Mortalidade do Brasil (1996/2001). Cad Saúde Pública 2006; 22:673-84.

29. Ministério da Saúde. Portaria GM nº 3.947 de 25 de novembro de 1998. Trata da compatibilização de Sistemas de Informação Saúde de base nacional. Diário Oficial da União 1998; 26 nov.
30. Bottari CMS. Câncer cérvico-uterino como condição marcadora: uma proposta de avaliação da atenção básica [Dissertação de Mestrado]. Rio de Janeiro: Escola Nacional de Saúde Pública, Fundação Oswaldo Cruz; 2007.

31. Divisão de Atenção Oncológica, Coordenação de Prevenção e Vigilância, Instituto Nacional de Câncer. Documento preliminar sobre indicadores de monitoramento das ações de controle do câncer de colo do útero no Brasil. Versão preliminar completa. Rio de Janeiro: Instituto Nacional de Câncer; 2005.

32. Secretaria de Políticas Públicas de Saúde. Plano de reorganização da atenção à hipertensão arterial e ao diabetes mellitus. Brasília: Ministério da Saúde; 2002.

33. Departamento de Informática do SUS. HiperDia - sistema de cadastramento e acompanhamento de hipertensos e diabéticos. Manual de operação. versão 1.5 M 03. Rio de Janeiro: Departamento de Informática do SUS; 2002.

34. PRO-ADESS: Metodologia de avaliação do desempenho do sistema de saúde brasileiro. http:// www.proadess.cict.fiocruz.br/ (acessado em 12/ Set/2006)

35. Starfield B. Atenção primária: equilíbrio entre necessidades de saúde, serviços e tecnologia. 2a Ed. Brasília: Organização das Nações Unidas para a Educação, a Ciência e a Cultura; 2002.

Recebido em 14/Mai/2007

Versão final reapresentada em 26/Fev/2008 Aprovado em 05/Mar/2008 